\title{
MENTAL STATE REPRESENTATION: SPATIOTEMPORAL CHARACTERISTICS
}

\author{
Alexander Oktyabrinovich Prokhorov \\ Kazan (Volga Region) Federal University, 11/10 Khalturina Str., Apt. 57, Kazan, 420032, Russia
}

Received 2014-02-13; Revised 2014-02-19; Accepted 2014-03-15

\begin{abstract}
Since the time of statement of the problem of states in psychology, the study of "sensuous" tissue - the mental state representation-takes a fundamental meaning. The problem is concluded in the following questions: "How is mental state represented in the consciousness of an individual?", "What is the specificity of the mental state representation as distinguished from the subject-matter representation?", "What are the mechanisms of the mental state representation occurrence and the peculiarities of its dynamics? The study of the mental state representation will allow to explain its specificity and difference from the figurative representation, the peculiarities of state explication as a representation in the consciousness and its relation with other elements of consciousness, will allow to show the regularities of the mental state representation development and its dynamics, factors, which influence the specificity of its occurrence, the regulatory role of the state representation in the vital function. From these perspectives, the article presents the results of the study of spatiotemporal characteristics of the mental state representation; reveals the peculiar features of the spatiotemporal organization of mental state representations: Relieves, specificity, magnitude, variability of indicators, changes of structural characteristics in time spans; considers the age-specific peculiar features of the spatiotemporal organization of mental state representations in terms of organization, stability, coherence and differentiated nature of spatiotemporal structures with the representatives of certain age groups.
\end{abstract}

Keywords: Mental State Representation, Space, Time, Structure

\section{INTRODUCTION}

The study of relations between the categories of mental phenomena is the most important methodological problem of psychology. Therefore, the study of the existential layer of consciousness, the "sensuous" tissue (according to A.N. Leontyev)-the mental state representation-takes a fundamental meaning.

Successful efforts of studying the mental state representation and the role of the representation in the context of the state self-regulation were made by (Dikaya, 2003). According to her, the representation structure includes the quasi-perceptual (representation in a qualified sense), psychophysiological (somatic) and semantic (interpretation, the concept of representation) and reflexive representation. The state representation is generated by the conditions, the subject matter of the activity and at the same time by internal feelings and the self-esteem. At the same time, the mental state representation is always timely and exists "here and now". The human memory simultaneously contains the representation of oneself in certain situations in the past and, by analogy, the representation of the needed future. Dikaya (2003) concluded that the mental state representation had a series of properties, which were typical of perceptive representation of the outworld, such as integrity, integrality and procedurality (Dikaya and Semikin, 1991).

At the study of representation, we take the following concepts as the basis. To our opinion, mental state representation is associated with sensory-perceptive processes (impression, feeling, perception), with the structures of personalized experience coupled with the perceptions (secondary representations) and memory, as well as with experiences and reflexion (Fakhrutdinova, 2009). The reality of mental state of an individual is provided by the experiences based on feelings and reflexion (Prokhorov and Fakhrutdinova, 2008; Prokhorov, 2009). Experiences determine and reinforce 
the mental representation of state, the intensity (vividness) of its display, where reflexion sets the contour of a representation, its proximity to and consistence with the actually experienced state.

According to these concepts, the following mechanisms cause the occurrence and reinforcement of the mental state representation. Internal feelings and impressions caused by events and situations experienced by an individual while passing the stage of comparing with the previous experience turn into ideas of the experienced state and then, through the process of realization to its representation. Similarly to the manner the subject representation appears and reinforces in the course of perception, the mental state representation is recorded and reinforced in the structures of memory during experiencing this state by the individual, thus forming the subjective experience.

From these perspectives, the state representation can be treated as the mental representation of the process of reflection and, as the result, as the unit of experience description within the worldview. Such an approach is typical of researchers of the procedural and dynamic aspect of mental representations and their cognitive functions (Cooper, 1990; Blatt et al., 1997; Kemp, 1998; Geller et al., 2010; Lukowitsky and Pincus, 2011). At the same time, mental representations are understood as internal structures formed during the course of human life, in which his worldview, as well as his view of the society and himself are represented (Bascoe et al., 2009; Savadori et al., 2001; Lotto et al., 2006). The mental state representation is a reflection of such an internal structure.

Further, the representation can be reproduced in actual vital processes in the form of an image, i.e., memory representation that is stored in the subjective experience (Gostev, 2007). This representation is not associated with any subject, it is a sensuous representation formed by the experience. In its turn, it can be enriched and modified in the course of vital processes like the representation image.

In terms of cognition, the state representation is a semantic space, which includes "accumulated" traces of experiences from previous activities, behavior, physiological reactions (Prokhorov, 2002). In the ontogenesis, the state representation changes to the stronger qualitative determinacy and amplification. The content of representation is the result of reflection of the accumulated experience of experiencing this state at various circumstances, situations and events, in which the individual was involved and is associated with his impressions, reflexive processes, experiencing peculiarities (Prokhorov, 2012a; 2012b).

The reflected elements of mental state are recorded by the consciousness in a certain combination, thus forming a structure. The latter is isomorphic to the real state. It is to be noted that subjective experience records the structures of space, time, rate of change (of motion) and intensity of mental state. Experience is projected on the actual own state. An individual perceives the characteristics of state from the perspective of behavioral, psychological, physiological and other indicators, gives a shape to this versatility, forms representation of this state and determines the quality. The individual structures the space of the state and creates a reference system, as space exists only when it is structured (Strelkov, 2001). A measure appears. Motion along one's "own scale" enables an individual to assess the space of the state, which is subjectively expressed as qualitative determinacy of certain elements, included in the state during experiencing the intensity of their display.

In this context, the subject matter of the subjective experience is a relatively stable spatiotemporal structure of state, experienced as a certain quality. Fixation of the structures of space, intensity, quality and modality of mental state in the experience in the form of representation is mediated by experiences and reflexive processes of the individual. The mechanism of such fixation was described in publications (Baddeley and Hitch, 1974; Kosslyn and Pomerantz, 1977).

According to these concepts, we studied the spatiotemporal characteristics of mental state representation.

\section{MATERIALS AND METHODS}

With the purpose of studying the spatial characteristics of the mental state representation, the tested persons described suggested states during various time spans representing their image. The representations of the following nonequilibrium states were assessed: Joy, anger (the states of high level of mental activity), calm (the state of medium level of mental activity), fatigue, loneliness (the states of low level of mental activity) within the following ranges: A minute, an hour, a day, a week, a month, half of a year, a year. I.e. representation of the same mental state was considered within the continuum between "one minute and one year". The research was carried out using the Relief of the Mental State questionnaire, in which 40 indicators of state were emphasized (Prokhorov, 1998). About 605 persons participated in the examination.

$697 \%$ took part in the study of the influence of age on the change of spatiotemporal characteristics of state representations. Age groups from 6-10 to 75-90 years of age were distinguished.

During the results processing, the average values and their variability were calculated and the correlation between the state indicators in each time span was computed ( 35 correlation matrices $40 \times 40$ were analyzed). The analysis of the state representations structures was 
carried out by indexes offered by (Karpov, 2004): The organization of a structure (the SOI) was calculated using the following formula: SOI (the Structure Organization Index $)=$ SCI+SDI, where SCI is the Structure Coherence Index, which was determined as a total of positive correlations with account of their importance. The relations significant on the $(\mathrm{p} \leq 0.05)$ level received the coefficient of 1 point; the relations significant on the $(p \leq 0.01)$ level received the coefficient of 2 points; the relations significant on the $(\mathrm{p} \leq 0.001)$ level received the coefficient of 3 points. SDI is the Structure Differentiation Index, which was determined in a similar manner, only the total of negative correlations was calculated.

\section{RESULTS}

Figure 1 represents the average values, which characterize the magnitude of representations of these states within the time spans between "one minute and one year". As the figure shows, the highest average values are typical of the joy state, slightly less - of the calm state and medium characteristics in all time spans are typical of fatigue.

When these data were analyzed, it was stated that the representation of each mental state was described with certain specificity of changes depending on the time span increase. For example, the typical feature of the joy state was the increase of the organization level and stability of relations accompanied by certain decrease of the magnitude. The calm state showed variability of the intensity and the level of the structure organization, as well as the stability of relations within the average values; they did not change much in different time spans. The fatigue state representation was described with the increase of the structure organization and stability of relations accompanied by the decrease of the intensity within the one-week and one-month time spans.

Let us consider to the variability indicators (Table 1). According to the table, the joy and calm states representations within each of the time spans demonstrate low variability of their indexes, i.e., these representations are the most stable ones over time if compared to the other states.

Taken as a whole, if we take all states, we can observe the growth of the indicators' variability over time, i.e. the stability of the representations structures becomes weaker within the longer time spans. The indicators of the fatigue representation show the highest variability, which increases over time. If compared to other structures, the fatigue representation has the weakest stability. Also, the values of variability of the anger and loneliness states representation are rather high.
Calculation of the Structure Organization Indexes (SOI) revealed the following peculiarities (Table 2). As evidenced by the table, the complicating of structures over time is typical of the high and low mental activity states representations (anger and fatigue). At the same time, the organization of the calm representation structure remains mainly unchanged within any time spans. The organization of the elements of the loneliness state structure has the following peculiar features: It increases in the oneweek and one-month time spans and decreases in the oneyear time span. The index of organization of the joy state representation structure is heterogeneous. The representation of the fatigue state maintains the highest level of the structure organization compared to the representations of other structures over all time spans.

It is to be noted that the increase of relations between the elements of the state representation over time is observed: Mental processes, physiological reactions, behavior and experiences, which ensures the integrity and the stability of the representation structure. The high index of organization also evidences the relatedness of the representation structure. This allows maintaining qualitative identity of the state over different time spans, as the stability of the representation structure determines its strength as an integral unit. To our opinion, the growth of the organization index is explained by the enriching of the states' representations as a consequence of their experiencing in the course of individual's vital function. Therefore, despite the change of indicators over time, the subjective identification of the same state is not affected.

The study of the influence of age on the change of spatiotemporal characteristics of mental state representations allowed revealing their specificity in various age groups. During the research, it was defined that all indicators of representations of positive states of the high level of activity (freshness, joy) except for the behavior indicators are characterized by the tendency to the decrease of intensity of their characteristics with aging and the approaching of the average level of values. The representations of the negative states of the high level of activity (anxiety) in the age dynamics demonstrate an expressed spread in values; they do not change much in ontogenesis. The values of representations of the states of the medium level of activity (equilibrium states-interest, calm) have insignificant spread of values of all state indicators. Their intensity insignificantly decreases with aging. The characteristics of the representations of the states of low level of activity (grief, tiredness, sorrow, fatigue) vary within the margins of lowered values, thus demonstrating high dispersion of all indicators with the intensity almost unchanged. 


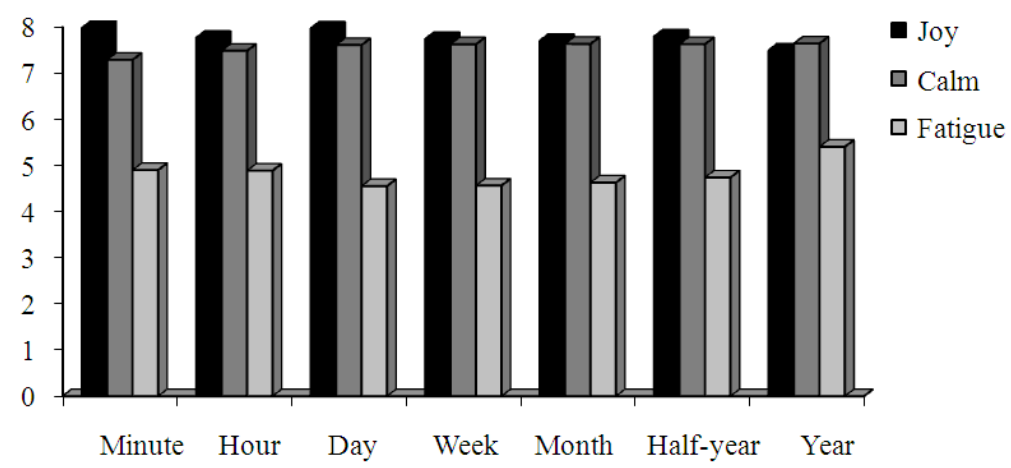

Fig. 1. Average values of the magnitude of the joy, calm and fatigue states within the time spans between "one minute and one year

Table 1. Ratios of variation of mental state representations within the "one minute-one year" time spans

\begin{tabular}{llllllll}
\hline & Minute & Hour & Day & Week & Month & Half of a year & Year \\
\hline Joy & 16.0 & 16.75 & 15.00 & 15.00 & 15.75 & 17.25 & 16.75 \\
Anger & 20.5 & 24.25 & 26.25 & 31.50 & 33.25 & 34.25 & 34.50 \\
Calm & 16.0 & 17.75 & 17.75 & 18.50 & 17.75 & 17.50 & 17.50 \\
Fatigue & 29.5 & 32.25 & 37.25 & 42.25 & 42.25 & 45.50 & 39.75 \\
Loneliness & 27.0 & 27.00 & 30.50 & 32.25 & 33.00 & 33.25 & 32.25 \\
\hline
\end{tabular}

Note: The values are expressed as percentage

Table 2. Values of the Structure Organization Indexes (SOI) of mental state representations within the "one minute - one year" time spans

\begin{tabular}{llllllll}
\hline & Minute & Hour & Day & Week & Month & Half-year & Year \\
\hline Joy & 907 & 1,503 & 990 & 1,213 & 1,240 & 1,115 & 1,376 \\
Anger & 860 & 743 & 1,365 & 1,574 & 1,708 & 1,744 & 1,598 \\
Calm & 1,176 & 1,264 & 1,298 & 1,167 & 1,258 & 1,089 & 1,224 \\
Fatigue & 1,613 & 1,725 & 2,298 & 2,207 & 2,294 & 2,269 & 2,230 \\
Loneliness & 948 & 1,281 & 1,253 & 1,641 & 1,633 & 1,413 & 1,458 \\
\hline
\end{tabular}

Table 3. The values of indexes of the mental state representation structure organization in various age groups

\begin{tabular}{lrlllrrrrrrr}
\hline & $6-10$ & $10-11$ & $11-13$ & $14-15$ & $15-17$ & $17-21$ & $21-30$ & $30-40$ & $40-60$ & $60-75$ & $75-90$ \\
\hline Buo. & 787 & & 308 & & 308 & 285 & 207 & 550 & 991 & 726 & \\
Joy & 786 & 392 & 769 & 453 & 1692 & 792 & 1443 & 1326 & 1493 & 1429 & 1602 \\
Anx. & 154 & 160 & 347 & 210 & 373 & 611 & 312 & 287 & 714 & 716 & 204 \\
Int. & 150 & & 913 & & 219 & 59 & 532 & & 464 & 334 & 66 \\
Calm & 97 & 138 & 561 & 578 & 251 & 169 & 510 & 453 & 434 & 334 \\
Grief & & & 247 & & 676 & 58 & 350 & 214 & 449 & 597 & 123 \\
Tired. & & & & & 184 & 253 & 226 & 204 & & 248 & \\
Sor. & 353 & 282 & 681 & 247 & 660 & 770 & 420 & 913 & 1413 & 1036 & 1176 \\
Fat. & 338 & 670 & 1060 & 589 & 1332 & 1020 & 836 & 405 & 520 & 1177 & 423 \\
\hline
\end{tabular}

Designation: Horizontally-Age groups; vertically-mental states. Buo.-buoyancy; Joy-joy; Anx.-anxiety, Int.-interest; Calm-calm; Grief-grief; Tired. -tiredness; Sor.-sorrow; Fat.-fatigue

As for the organization of structures, the most interconnected structure of representation of the state of high level of activity is typical of joy in the age groups of 15-17 and 75-90 years of age (Table 3). The buoyancy state has high values of organization in the 6-10 and 40-60 age groups. The representation of the negative state of high level of mental activity (anxiety) shows high values of organization in the 17-21, 40-60 and 60-75 age groups.
The indicators of representation of the states of medium level of mental activity (interest, calm) are characterized with the organization growth within the range of 11-13-21-30 years of age and its decrease at older age. Each mental state of the low level of mental activity demonstrates certain age-related specificity. The indicators of organization of representations of the grief, sorrow, tiredness and fatigue states are heterogeneous 
and demonstrate expressed spread in values. At the same time, it is to be noted that sorrow increases with aging, which is probably related to the frequency of experiencing this state. The high fatigue organization is typical of the 15-17 and 60-75 age groups.

\section{DISCUSSION}

When addressing the results of the mental state representation study, we need to note the following. The considered characteristics of representation: Phenomenological peculiarities, structure, changes of the representation within different time spans, age-related specificity and others, allow treating the state representation as a relatively stable establishment, which integrates various psychological, physiological and behavioral indicators. These results are confirmed by the researches of the mental state representation structure (Artishcheva, 2012a). The representation characteristics are quite comprehensively realized by an individual, which manifests itself in the assessments and descriptions of the tested persons. The researches of the figurative level of the mental state representation carried out in (Alexeeva, 2012) also evidence this. They also show that it is possible to actualize the concept of the state itself through actualization of individual concepts of a situation, of which a certain mental state is typical. Depending on the situations, the concepts of mental states are reinforced based on the human experience in experiencing certain states in various circumstances.

But it is not a subject representation; it is a consciousness representation, a "vision", which can be determined as a total of perceptive characteristics reflecting in the form of a structured combination the psychological, somatic, behavioral and other indicators of an individual, isomorphic to the experienced mental state. The mental state representation is reflected in the consciousness in the form of the experienced configuration, interrelated subjective and objective scales, which occur following the actualized state. Stability and integrity of representation are relative and depend on a series of variables: The energetic component of a state, the time length, sign, modality of the state, subject matter and other characteristics. Stability and variability of a representation in the time spans as shown by Artishcheva in her researches (Artishcheva, 2012b; 2013) are probably the reflection of the procedurality of the representation as well as the heterogeneity of the processes of adaptation of an individual to the circumstances and the situations existent.
Further research of interrelations of the state representation with the consciousness components: Conceptual characteristics, reflexion, experiences, cognitive processes, subjective experience and others, as well as with personally subjective characteristics depending on vital function situations and events, will allow to define the phenomenon of mental state representation more comprehensively in the context of mental phenomena and mental organization of an individual.

\section{CONCLUSION}

The considered characteristics of representation allow treating state representation as a relatively stable establishment. They are quite comprehensively realized by an individual, which manifests itself in the assessments and descriptions of the tested persons. To our opinion, the stability of representation is associated with the subjective experiencing and, first of all, with the frequency, length and intensity of its actualization in certain situations of the individual's life activity. Consequently, certain elements are reinforced in the representation structure.

The following phenomenological peculiarities of the spatiotemporal organization of mental state representations have been considered: Specificity and magnitude of their displays, variability of indexes, changes of structural characteristics within time spans, organization and stability. It has been stated that mental state representations are characterized with the tendency of changing if the time spans are increased, while the subjective identification of a state remains unchanged.

The age-specific peculiarities of the spatiotemporal organization of mental state representations have been revealed. The specificity of mental state representations of various level of mental activity has been stated in terms of organization, coherence and differentiation of the spatiotemporal structures with the representatives of certain age groups. It was shown that, in terms of age, the changes of spatiotemporal structures are characterized by wave dynamics.

Credits. The article was written as part of the grant of the Russian State Scientific Fund, project \# 10-0600074a.

\section{REFERENCES}

Alexeeva, E.M., 2012. Study of the representational level of mental state representation. Proceedings of the 5th International Conference of Cognitive Science, (CS ‘12), Kaliningrad, Russia, pp: 211-213. 
Artishcheva, L.V., 2012a. Representation of mental state: Structural aspect. Proceedings of the International Scientific and Practical Conference "Modern Reality and the Life Path of a Human: Psychological Aspects", (PA' 12), TulGU, Tula, pp: 296-300.

Artishcheva, L.V., 2012b. Stability-variability of structures of mental state representations in the continuum. Proceedings of the 6th Winter School of Mental States Psychology Psychology of Mental States, (PMS '12), Kazan University, Kazan, pp: 2024.

Artishcheva, L.V., 2013. Dynamic Characteristics of Mental State Representations. In: Psychology of Mental States, Prokhorov, A.O. (Ed)., Kazan: Kazan University, pp: 22-34.

Baddeley, A.D. and G. Hitch, 1974. Working memory. The psychology of learning and motivation. Adv. Res. Theory, 8: 47-89. DOI: 10.1016/S00797421(08)60452-1

Bascoe, S.M., P.T. Davies, M.L. Sturge and E.M. Cummings, 2009. Children's representations of family relationships. Dev. Psychol., 45: 1740-1751. DOI: $10.1037 / \mathrm{a} 0016688$

Blatt, S.J., J.S. Auerbach and K.N. Levy, 1997. Mental representations in personality development, psychopathology and the therapeutic process. Rev. General Psychol., 1: 351-374. DOI: 10.1037/10892680.1.4.351

Cooper, L.A., 1990. Mental representation of threedimensional objects in visual problem solving and recognition. J. Exp. Psychol., 16: 1097-1106. DOI: 10.1037/0278-7393.16.6.1097

Dikaya, L.G. and V.V. Semikin, 1991. The regulating role of the functional state in extreme operational environment. Psychol. J., 12: 55-65.

Dikaya, L.G., 2003. Mental Self-Regulation of the Human Functional State. 1st Edn., Institute of Psychology of RAS, Moscow, pp: 318.

Fakhrutdinova, L.R., 2009. Theory of Experiences. 1st Edn., Kazan State University, Kazan, pp: 418.

Geller, J.D., B.A. Farber and C.E. Schaffer, 2010. Representations of the supervisory dialogue and the development of psychotherapists. Psychotherapy Theory Res. Pract. Train., 47: 211-220. DOI: 10.1037/a0019785

Gostev, A.A., 2007. Psychology of Secondary Representation. Moscow. 1st Edn., Institute of Psychology of RAS, pp: 512.
Karpov, A.V., 2004. Psychology of Reflexive Mechanisms of Activity. 1st Edn., Institute of Psychology of RAS, Moscow, pp: 421.

Kemp, S., 1998. Medieval theories of mental representation. History Psychol., 1: 275-288. DOI: 10.1037/1093-4510.1.4.275

Kosslyn, S.M. and J.R. Pomerantz, 1977. Imagery, propositions and the form of internal representation. Cognitive Psychol., 9: 52-76. DOI: 10.1016/00100285(77)90004-4

Lotto, L., E. Rubaltelli, R. Rumiati and L. Savadori, 2006. Mental representation of money in experts and nonexperts after the introduction of the Euro. Eur. Psychol., 11: 277-288. DOI: 10.1027/10169040.11.4.277

Lukowitsky, M.R. and A.L. Pincus, 2011. The Pantheoretical nature of mental representations. Psychoanalytic Psychol., 28: 48-74. DOI: 10.1037/a0020849

Prokhorov, A.O. and L.R. Fakhrutdinova, 2008. About the relation between experiences and mental states. Humanities, 150: 50-56.

Prokhorov, A.O., 1998. Psychology of Nonequilibrium States. 1st Edn., Institute of Psychology of RAS, Moscow, pp: 152.

Prokhorov, A.O., 2002. Semantic Spaces of Mental States. 1st Edn., Fenix, Dubna, pp: 280.

Prokhorov, A.O., 2009. Conceptual Regulation of Mental States. 1st Edn., Institute of Psychology of RAS, Moscow, pp: 352.

Prokhorov, A.O., 2012a. Representation of mental state: Mechanisms and regularities. Proceedings of the 5th International Conference of Cognitive Science, (CCS’ 12), Kaliningrad, Russia, pp: 590-591.

Prokhorov, A.O., 2012b. Representation of Mental State: Phenomenological Peculiarities. In: Issues of Social Psychology, Strakhov, V.I. (Ed.). Saratov: Nauka, pp: 39-51.

Savadori, L., E. Nicotra, R. Rumiati and R. Tamborini, 2001. Mental representation of economic crisis in italian and swiss samples. Swiss J. Psychol., 60: 1114. DOI: $10.1024 / / 1421-0185.60 .1 .11$

Strelkov, Y.K., 2001. Engineering and Professional Psychology. 1st Edn., Academy, Moscow, pp: 360. 